\title{
Wireless controller and smartphone based in- teraction system for electric bicycles.
} Jorge Revuelta ${ }^{a}$, Gabriel Villarrubia ${ }^{a}$, Alberto L. Barriuso ${ }^{a}$, Daniel Hernández ${ }^{\mathrm{a}}$, Álvaro Lozano ${ }^{\mathrm{a}}$, Marco Antonio de la Serna González ${ }^{\mathrm{b}}$

\author{
jrevuelta@acm.org , gvg@acm.org ${ }^{\mathrm{a}}$, albarriuso@acm.org ${ }^{\mathrm{a}}$, murciego@acm.org ${ }^{\mathrm{a}}$, ahernandezdelaiglesia@acm.org \\ maserna@stagemotion.com ${ }^{\mathrm{b}}$ \\ ${ }^{\text {a }}$ ACM Student Member \\ ${ }^{b}$ EBIKEMOTION® TECHNOLOGIES S.L.
}

KEYWORD

Wireless controller; smartphone; electric bicycle; ebikemotion

\begin{abstract}
Throughout the last years, great improvements have been made in the automotive industry, a sector with a high impact in both economics and social environments. Many development efforts have targeted on satisfying the need of providing smart environments to the end user in vehicles, such as cars. This paper proposes a brand-new solution in the automotive field, consisting of a new product family which aims to transform the traditional bicycle to an electric bicycle using an architecture that provides an adaptive environment to the user, improving the driving experience enabling value-added services.
\end{abstract}

\section{Introduction}

Bicycle are vehicles with a tremendous importance throughout history in our society, combining the advantages of a personal vehicle of small size with lowering emissions and promoting sports and physical exercise. The bike design has been altered in recent years with the development of accessories such as motors and batteries that turn it into an electric vehicle. In recent years there has been a gradual migration in the technological development of urban mobility, focusing on the development and expansion of the electric bicycle (Cherry, Yang, Jones, \& He, 2016)(Astegiano, Tampère, \& Beckx, 2015). Nowadays, companies involved in bicycle and automobile sector, are investing in the production of new models of bikes, with lower production costs that make the number of sales grow each month. We can highlight that the bicycle market is continuously growing, being competition between companies higher every moment. The key reason for the growth in the number of sales is due to the widespread growth of urban centers (Ling, Cherry, Yang, \& Jones, 2015), where the motor vehicle takes the lead due to the agile development of transport infrastructure (Omatu et al., 2014). Another reason lies in the pollution generated by the user of hydrocarbon-based vehicles (Abagnale et al., 2015), where electric ones take center stage but high powered are at the very early stage of development yet.

Electric bikes are in the process of expansion, attracting the attention of researchers and manufacturers (Du et al., 2013). Nowadays, one of the aspects that needs attention and is an effort to design is the development of robust and flexible in terms of hardware, software and communications architectures. Likewise it is important to identify a model of integration for all these elements that will allow building a system of 
interaction and control between the user and the electric vehicle (Wong \& Huang, 2013). Especially, it is of particular interest the design of systems for the active integration of users in the system, optimizing the performance of all components through algorithms and systems using new communication technologies (geolocation, telephony, mobile messaging, etc.).

To achieve this objective, we propose define and design a comprehensive control system and a wireless mechanism (joystick) of human-machine interaction.

The development of a flexible and open architecture will allow the gathering of information about the user, road and environment, being able to detect unusual situations such as accidents or theft. The architecture in this article will serve as a basis for a future where users can share experiences and racing with other users without direct vision between cyclists. Information about physical phenomena such as wind, dangerous zones, etc., will be able to be gathered in real time. In addition, the state of the system, like battery status and motor faults, should be monitored so that the user has a comprehensive control of the vehicle and its environment.

This article is organized as follows: section 2 shows a review about the state of the art and related projects, section 3 describes the architecture proposal, section 4 describes a case study, which will be used to evaluate the system, and finally section 5 exposes the results and conclusions obtained.

\section{Background}

Currently on the market exists a wide range of integrated systems for the management and monitoring of electric bicycles. Most of these systems are composed of several modules interconnected together via standard protocols, both wired and unwired.

For reading and processing data and information coming through the own electric bicycle (voltage, amperage, instantaneous power consumption, etc.) are included with the installation on the bike of a series of reading sensors that send, through a single bus, all the information in real time.

For sending information, as described in (Ismail, Muharam, \& Pratama, 2015), the CAN-Bus (Control Area Network) standard is used which enables hardware devices (microcontrollers) to communicate with each other without the need for a computing unit core, as shown in Fig 1, which due to its inherent characteristics it has been shown to be an efficient and reliable method for exchanging real-time data in a system (Xia, Zhang, Bai, \& Xue, 2013).

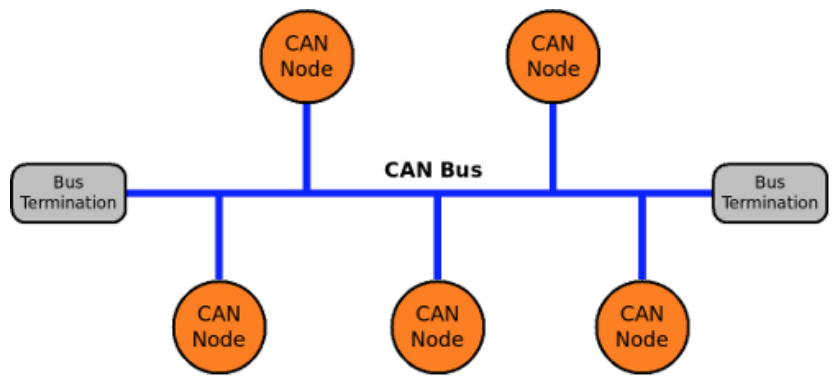

Fig 1. Architecture of a CAN-Bus.

This protocol was originally introduced in 1983 by Bosch and later was approved as an ISO standard (ISO11898 and various revisions and subsequent variants).

This protocol is already widely used in vehicles for communication between devices and the features described above make it ideal for a bike to have sensors that are transparent and are abstracted from the hardware to which they are connected by sending the system's relevant information without worrying about the hardware.

The architecture that make up these CAN-Bus enabled systems are usually of two main types: 
Centralized: the system counts on a central hardware device that collects information on a single node which can be active if it performs processing and interpretations of the information received; or passive, if its role is gather the information and forward it somewhere else.

Decentralized: these systems have a single bus which carries the information without worrying about where it's going or what is going to be done with it.

The centralized architecture offers advantages over decentralized related to the capability to process the information in a real time basis which allows the system to make decisions without the need for additional equipment in the architecture, as well as adding new devices with new bus that are connected to the central node, as well as the independence between the different elements of the system. However, the main disadvantage is the dependence of the entire system on the hub or central node, as otherwise the system would be isolated.

The limitation of this type of implementation is its closed and wired nature of the communication of the integrated devices in the system. The popularization of smartphones and its low cost with increasingly higher computing capabilities and wireless communication (Lee \& Lee, 2014) make it suitable of being the bridge element between the wired system which conforms the electric vehicle itself with the outside. Due to the characteristics of the system to which we refer, low power consumption and medium range communication properties become keys to communicate the vehicle system with foreign elements. That is why Bluetooth becomes the focus for communication devices interconnected by CAN-Bus with an external device outside the vehicle system itself.

Both 2.0 and 3.0 versions of the Bluetooth protocol (Cho, Park, Cho, Seo, \& Han, 2015) was designed for the data transmission to low range but at high rate, without being possible to operate the protocol and the hardware in a low power mode until the Bluetooth Low Energy (BLE) was introduced in the 4.0 protocol update. This review focused on the operation in situations of very low consumption at lower rate and the possibility of data failures in the transmission.

The low cost of integrating such hardware into existing CAN-Bus systems have made major manufacturing companies have integrated accessories and ebikes such as Bosch and COBI, as can be shown in Fig 2.

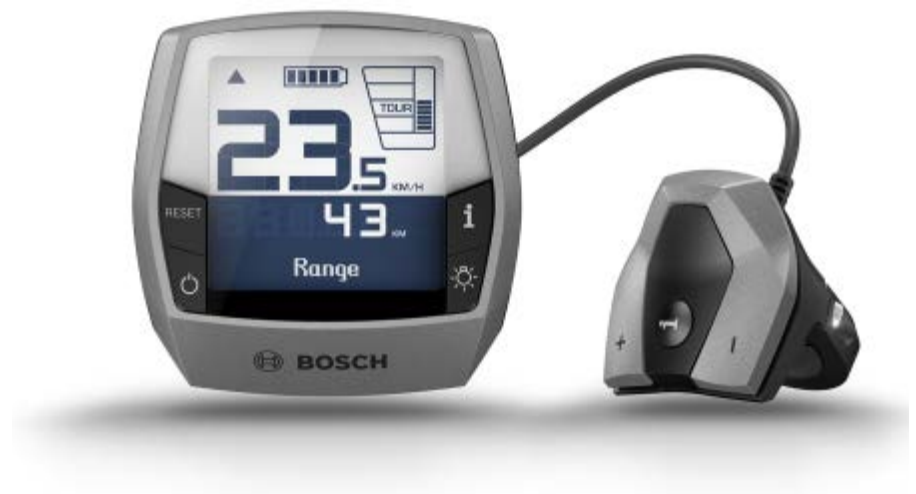

Fig 2. Bosch information display and joystick to hands-free manipulation on operation.

The main problem which faces the integration of the solutions proposed by different companies is the proprietary communication protocols and the requirement of its solutions for interaction throughout the 
vehicle system. Besides these closed systems include the capability of pairing with a smartphone via Bluetooth BLE, restricting the user experience to the tool provided by the brand.

In this paper we propose the use of a central hardware device that can be used with existing CAN-Bus systems for the communication of the system devices and its external communication through an open protocol Bluetooth BLE based, but also a software system for both iOS and Android that offers a customizable user experience in response to the needs of users of the systems.

On the other hand, there is a need for the presence of an element of human-computer interaction to provide the user the ability of modify, make decisions and control the system status. The majority of market systems have a main element of interaction like a joystick, as shown in Fig 3.

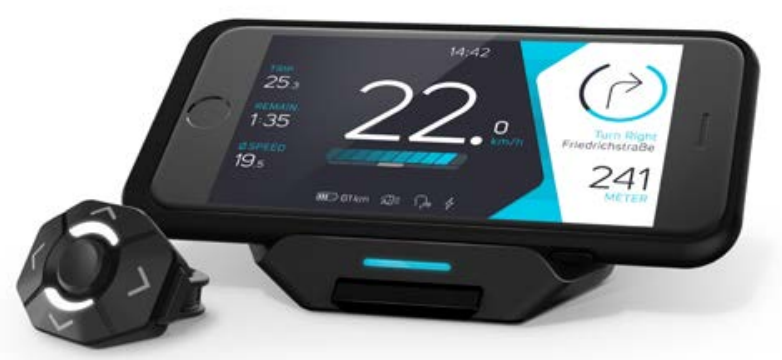

Fig 3. Example of a human-computer interaction device for electric bicycles.

The joystick is a hardware device connected to the electric bicycle system and, by operating the integrated controls, allows the user to set the system parameters and interact with it.

These interactive elements have similar problems of integration as the CAN-Bus ones, its closed protocol and the inability to adapt the user experience to the needs of each individual. Therefore, in this article, also a hardware device is proposed, that enables the integration of an interface with the rest of the system through human interaction that is capable of operate with most of CAN-Bus based systems applied to electric bicycles.

\section{Proposal}

This sections describes the architecture that connects universally an accessory to control the bicycle turning it into an electric vehicle (Villarrubia, De Paz, Bajo, \& Corchado, 2014).

The realization of the idea proposed is proposed by means of an architecture has shown in Fig $\mathbf{4}$ and whose components are described below:

- Human Communication Interface.

- $\quad$ EBM Communication Module - Gateway.

- Intelligent System for Social Computing.

- Joystick.

In the Fig 4, a component diagram that conforms the architecture is shown. It includes the wireless communication between the Communication Module (EBM - Gateway) and the Human Communication Interface as well as the different wired connection between each installed reading device in the electric vehicle with the central node. Every communication of the system with the external devices is made through Bluetooth BLE and the proposed protocol. 


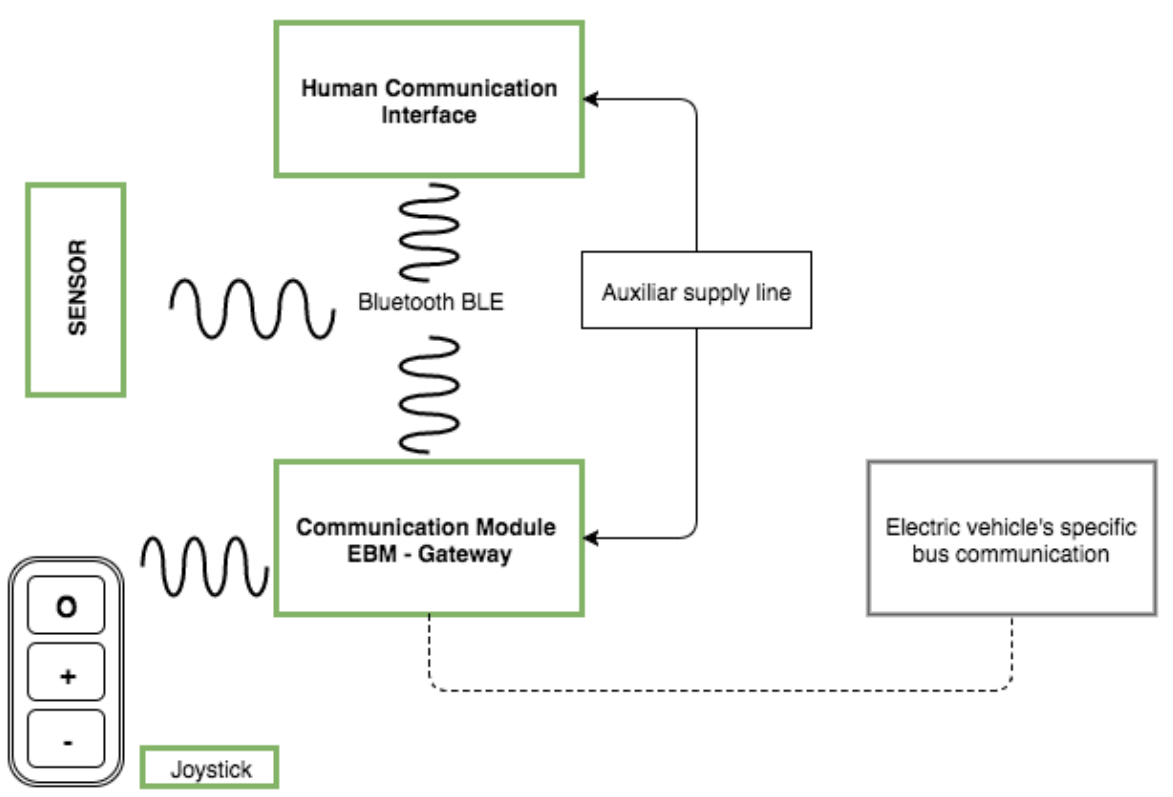

Fig 4. Architecture of the proposed platform.

Control System / EBM Gateway: The aim of the proposed system is to provide an effective and efficient management of an electric vehicle (bicycle) and adapted to the need of the user through a humanmachine interface incorporating innovative features as context awareness. It provides a new design which incorporates a communication module EBM (Bluetooth) which performs as gateway between the electric motor and the human communication interface (and additional devices that may be connected).

Joystick: A differential architecture aspect in the introduction of a mechanism of interaction with the user based on a joystick. Although nowadays joysticks can be found in science and market that allows the user to interact with the bicycles, you cannot find a joystick like the one proposed in this project: wireless, symmetrical (for both left-handed and right-handed users) that allows an adaptive mapping functions of the power unit of the vehicle.

The proposed architecture aims to provide the system with communication capabilities through the smartphones available in the market, so it can monitor the information from the engine, set its parameters (wheel size or battery type and number of cells) and put them combined with context sensitive and social information. Such information can be obtained through sensors such as GPS or advanced calculations from third-party computers connected by Bluetooth BLE (pulse bands, clocks, etc.). 


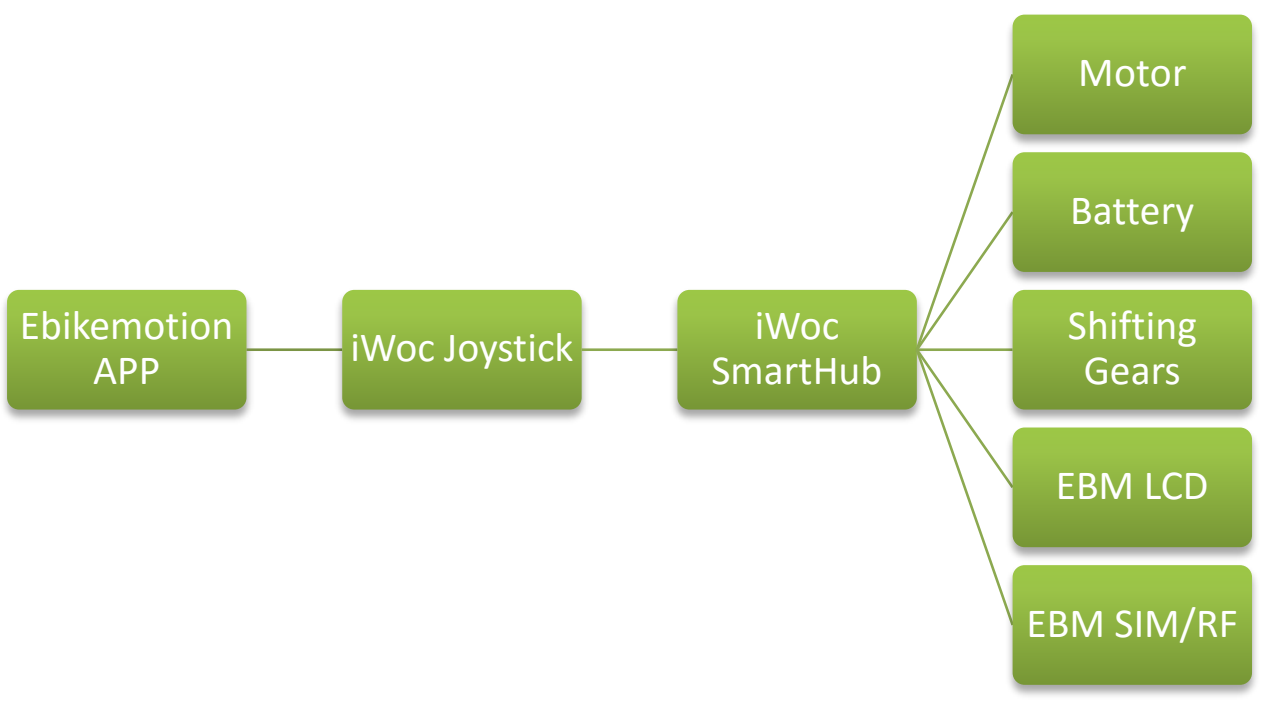

Fig 5. Proposed communication between devices.

To fetch the engine information, the human communication interface receives several information chunks of information related to the battery or the motor status through the Bluetooth interface in the user's communication device (typically a smartphone), as shown in Fig 5, so there will be a hardware device that may be connected to the serial bus engine and will pack and send the information to the human communication interface through a standard protocol. Also it makes necessary to exist the design and construction of a physical interaction device such as the joystick, which guarantees the user's capability to interact with the system without taking its hands off the handlebars.

The communication protocol proposed is based on the BLE's own characteristics. The system emits periodic messages, as shown in Table 1, without confirmation and around a $2 \mathrm{~Hz}$ rate each one, with the state of each one of the components that are connected to the system. The creation of the communication protocol of message exchange is accompanied by a software system precompiled that remain available for most mobile platforms (Android and iOS), allows the interpretation and synthesizing information provided by the system and allows developers perform actions (show and hide information) and decisionmaking through the communication interface.

\begin{tabular}{|ll|}
\hline Message & Action \\
\hline 0x01\#bYX\#@ & Button pulsation \\
\hline \#IXY\#@ & LED status \\
\hline \$s\$A\#000000\#@ & Bike autonomy \\
\hline \$m\$T\#000\#@ & Engine temperature \\
\hline \$m\$V\#000\#@ & Instantaneous speed \\
\hline \$m\$P\#000\#@ & Instantaneous power \\
\hline
\end{tabular}

Table 1. Information exchanging protocol.

The fundamental component which conforms the human interaction interface is the development of a smartphone application supported in the architecture and services provided through the Bluetooth BLE and CAN-Bus communication systems. 
The proposed application has unprecedented modularity that allows full customization of the user experience form deciding the components of information visible on the screen at all times to maximize the user's attention in driving the bike and show the information relevant to each individual.

Also features offline operation, consistent with the nature of the common profile of mountain biker that can rarely have a reliable connection for browsing the internet for directions. For this reason, the application has the ability to install map packages that can be used offline, ensuring total user experience even in offline environments.

\section{Conclusions and future work}

The proposal exposed by this article are taken from different software and hardware products that together provide a driving experience management and interaction with an electric bike system that gives the user complete freedom of customization according to individual characteristics.

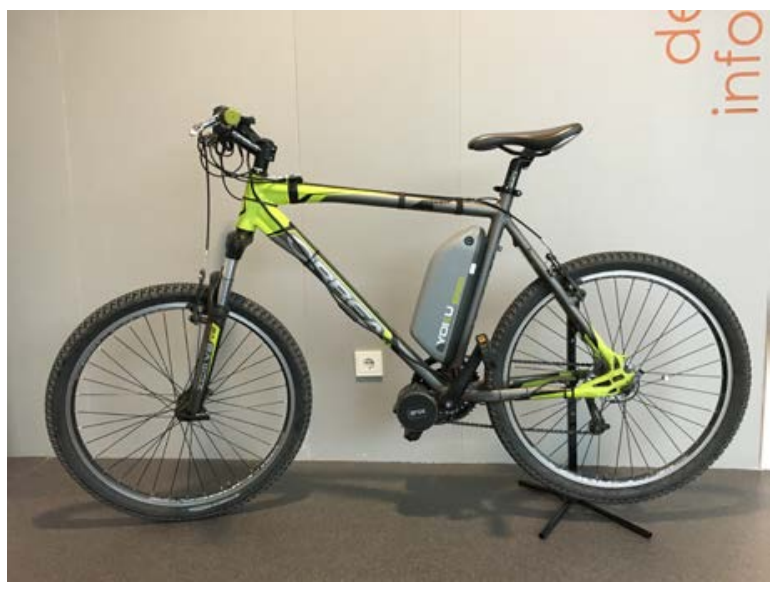

Fig 6. Shot of the electric bicycle.

To create the system and in order to prove that the system is open and fully compatible with devices already on the market a conventional bike brand Orbea was used to which was added a battery band Epro model Yoku YK3611ZDDR1 compatible with CAN-Bus protocol as well as a motor brand 8FUN, elements shown in Fig 6.

Both hardware elements CAN-Bus compatible were connected following a centralized architecture with a central node that has the Bluetooth BLE communication system that lets the user interact with external devices.

The main external device that the system can communicate with is an Android or iOS smartphone Bluetooth BLE compatible that have the application installed, the interface of which can be seen in Fig 7. This implementation achieves the goals of providing the user the ability to customize their experience on the bike in the most flexible way possible and provide services such as navigation with voice instructions through tracks and bike trails as well as installing maps of territories in offline mode so Internet connection is no longer required for the operation of the application. 


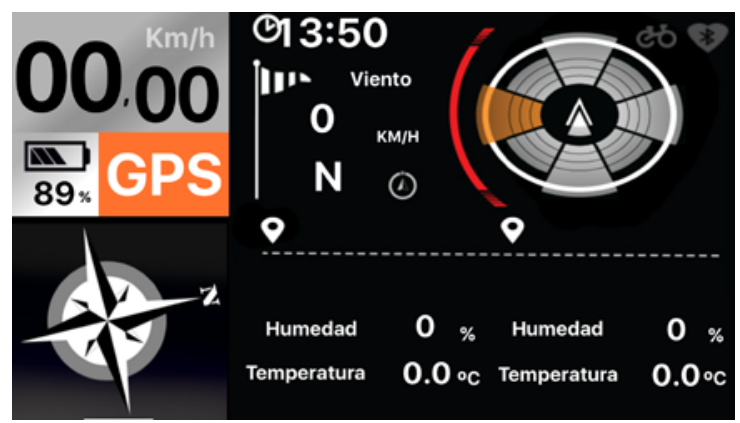

Fig 7. Fully customizable interface of the Ebikemotion app.

Finally, to address the objective of offering the user an interaction way with the system (including the smartphone itself) a human-machine interaction integrated hardware device has been designed, as shown in Fig 8, comprising a joystick that has three buttons for controlling with pulses of short and long term, different aspects of the behavior of both the electric bike (level of assistance, turning on and off the system, pairing mode) and the application (navigation between screens, repeat navigation instructions, spoken summary of the tracking, etc.).

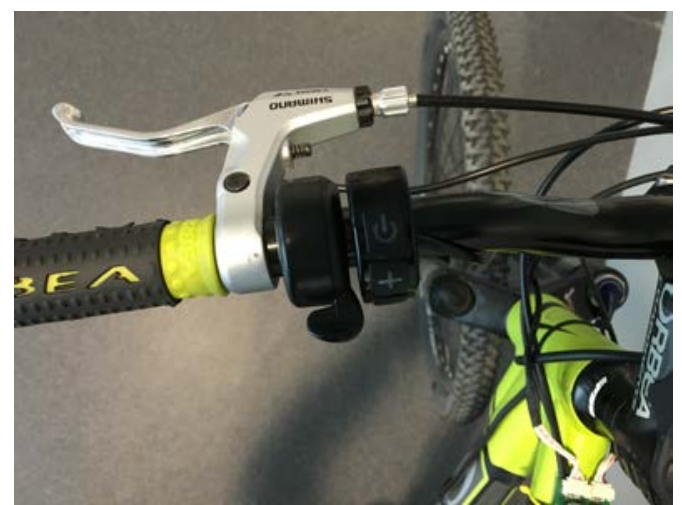

Fig 8. Human-machine interaction device proposed.

\section{Acknowledgements}

This work has been carried out by the project, GatEBike - Arquitectura basada en Computación Social para el Control Inteligente e Interacción en Bicicletas Eléctricas (RTC-2015-4171-4).

The project has been supported by European Commission and Ministerio de Economía y Competitividad (RetosColaboración 2015).

The research of Alberto L. Barriuso has been co-financed by the European Social Fund (Operational Programme 2014-2020 for Castilla y León, EDU/128/2015).

\section{References}

Abagnale, C., Cardone, M., Iodice, P., Strano, S., Terzo, M., \& Vorraro, G. (2015). A Dynamic Model for the Performance and Environmental Analysis of an Innovative e-bike. Energy Procedia, 81, 618627. http://doi.org/10.1016/j.egypro.2015.12.046

Astegiano, P., Tampère, C. M. J., \& Beckx, C. (2015). A Preliminary Analysis Over the Factors Related 
with the Possession of an Electric Bike. Transportation Research Procedia, 10, 393-402. http://doi.org/10.1016/j.trpro.2015.09.089

Cherry, C. R., Yang, H., Jones, L. R., \& He, M. (2016). Dynamics of electric bike ownership and use in Kunming, China. Transport Policy, 45, 127-135. http://doi.org/10.1016/j.tranpol.2015.09.007

Cho, K., Park, G., Cho, W., Seo, J., \& Han, K. (2015). Performance analysis of device discovery of Bluetooth Low Energy (BLE) networks. Computer Communications. http://doi.org/10.1016/j.comcom.2015.10.008

Du, W., Yang, J., Powis, B., Zheng, X., Ozanne-Smith, J., Bilston, L., \& Wu, M. (2013). Understanding on-road practices of electric bike riders: an observational study in a developed city of China. Accident; Analysis and Prevention, 59, 319-26. http://doi.org/10.1016/j.aap.2013.06.011

Ismail, K., Muharam, A., \& Pratama, M. (2015). Design of CAN Bus for Research Applications Purpose Hybrid Electric Vehicle Using ARM Microcontroller. Energy Procedia, 68, 288-296. http://doi.org/10.1016/j.egypro.2015.03.258

Lee, S., \& Lee, S. (2014). Early diffusion of smartphones in OECD and BRICS countries: An examination of the effects of platform competition and indirect network effects. Telematics and Informatics, 31(3), 345-355. http://doi.org/10.1016/j.tele.2013.12.002

Ling, Z., Cherry, C. R., Yang, H., \& Jones, L. R. (2015). From e-bike to car: A study on factors influencing motorization of e-bike users across China. Transportation Research Part D: Transport and Environment, 41, 50-63. http://doi.org/10.1016/j.trd.2015.09.012

Omatu, S., Bersini, H., Corchado, J. M., Rodríguez, S., Pawlewski, P., \& Bucciarelli, E. (Eds.). (2014). Distributed Computing and Artificial Intelligence, 11th International Conference (Vol. 290). Cham: Springer International Publishing. http://doi.org/10.1007/978-3-319-07593-8

Villarrubia, G., De Paz, J. F., Bajo, J., \& Corchado, J. M. (2014). Ambient agents: embedded agents for remote control and monitoring using the PANGEA platform. Sensors (Basel, Switzerland), 14(8), 13955-79. http://doi.org/10.3390/s140813955

Wong, J.-T., \& Huang, S.-H. (2013). Attention allocation patterns in naturalistic driving. Accident; Analysis and Prevention, 58, 140-7. http://doi.org/10.1016/j.aap.2013.04.033

Xia, J., Zhang, C., Bai, R., \& Xue, L. (2013). Real-time and reliability analysis of time-triggered CANbus. Chinese Journal of Aeronautics, 26(1), 171-178. http://doi.org/10.1016/j.cja.2012.12.017 